\title{
ATLAS-detector operations with beams: the data-acquisition perspective
}

\author{
R. Ferrari ${ }^{\text {* }}$ on behalf of the ATLAS TDAQ Group

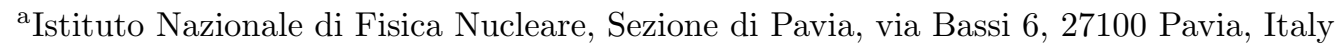

The ATLAS data acquisition system is based on about $2 \mathrm{k}$ processing nodes, interconnected by a multi-layer Gigabit Ethernet network. About 20k applications provide the needed capabilities in terms of run control, event selection, data flow, local storage and data monitoring.

The whole system has been successfully commissioned during the last two years with cosmic ray and calibration data and it turned out to be robust and reliable. Nevertheless, the continuous operation with beams, the concurrent trigger commissioning, and the understanding of detector and physics performance pose new challenges. The flexibility of the data acquisition infrastructure will be probed and exploited, in order to comply with unseen working conditions in terms of data flow, monitoring and configuration requirements. The goal is to minimise both downtime and dead-time, allowing for runtime reconfiguration of the data acquisition and sub-detectors systems as well as for automatic error handling and recovery.

\section{INTRODUCTION}

ATLAS[1] is one of the two general purpose experiments to exploit the physics discovery potential of the CERN Large Hadron Collider (LHC). At the nominal energy and luminosity of LHC, with a typical event size of $\sim 1.6 \mathrm{MB}$, the ATLAS detector would produce $64 \mathrm{~TB} / \mathrm{s}$ of mostly non-interesting data. The Trigger and Data AcQuisition (TDAQ) system [2] is designed to handle and reduce such throughput by several orders of magnitude without loosing physics potential.

\section{Trigger and Data Acquisition (TDAQ)}

The data acquisition system is based on a three-level trigger architecture (Fig. 1). The first (LVL1) is a hardware trigger, whereas the second (LVL2) and the event filter (EF), are based on software algorithms. LVL2 and EF form the High Level Trigger (HLT).

The LVL1 select events with a maximum latency of $2.5 \mu \mathrm{s}$, at a rate of $75 \mathrm{kHz}$. It uses coarse calorimeter and muon detector information to define Regions of Interest (RoI) and send them to the LVL2. An RoI is a slice of the detector readout, pointing to the collision vertex and surround-

\footnotetext{
*e-mail: roberto.ferrari@cern.ch
}

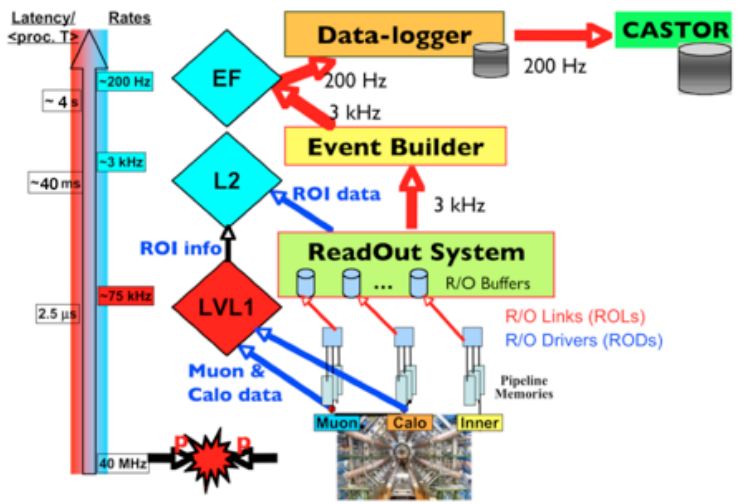

Figure 1. TDAQ architecture.

ing the LVL1 trigger source. The TDAQ system uses RoIs to reduce the data-flow requirements and to guide the HLT event selection. The raw event fragments from the frontend electronics get buffered in hardware pipelines and mostly dropped according to the LVL1 decision. Accepted events are partially assembled and collected in the 1600 readout buffers of the ReadOut computing System (ROS). The ROS nodes 
work in a push-pull mode: data are pushed into the input buffers, and pulled out (or deleted) on request from other subsystems.

The LVL2 receives the RoIs and the LVL1 result, gets the RoI data from the ROS system and performs a local, refined, analysis within the RoI. With $\sim 2 \%$ of the full information, in a mean processing time of $40 \mathrm{~ms}$, it reduces the rate down to $\sim 3 \mathrm{kHz}$, i.e. the throughput from $110 \mathrm{~GB} / \mathrm{s}$ to $4.5 \mathrm{~GB} / \mathrm{s}$. The usage of RoIs is an effective rejection mechanism which imposes less severe requirements on the system, in terms of both bandwidth and CPU needs.

The LVL2 decision is transferred to the event builder system (EB), where applications fully assemble the accepted events. A special EB feature is the so-called partial event building. Even during standard physics runs, events for subdetector calibration purposes are processed. Such events require limited sub-detector information, depending on the specific calibration needs. The reduced size for calibration events allows for a higher LVL2 accept rate, while keeping the total throughput constant.

Fig. 2 shows that the EB has been successfully validated during test runs in 2009. The performance scales linearly with the number of applications, currently 94 over 63 nodes. The performance is limited by the installed EF network bandwidth of $6 \mathrm{~GB} / \mathrm{s}$, about half of the final system design but already beyond the design EB throughput.

The EF accesses the fully assembled events, operating the trigger menu on the full event data. The mean processing time is requested to be around $4 \mathrm{~s}$. The rate is finally reduced to $\sim 200 \mathrm{~Hz}$, corresponding to a throughput of $300 \mathrm{MB} / \mathrm{s}$. Accepted events are then sent to the Sub-Farm Output (SFO) nodes, split into streams, according to their trigger content, stored locally and finally moved to the CERN central mass storage facility. Events matching more streaming conditions get duplicated, thus increasing the overall throughput.

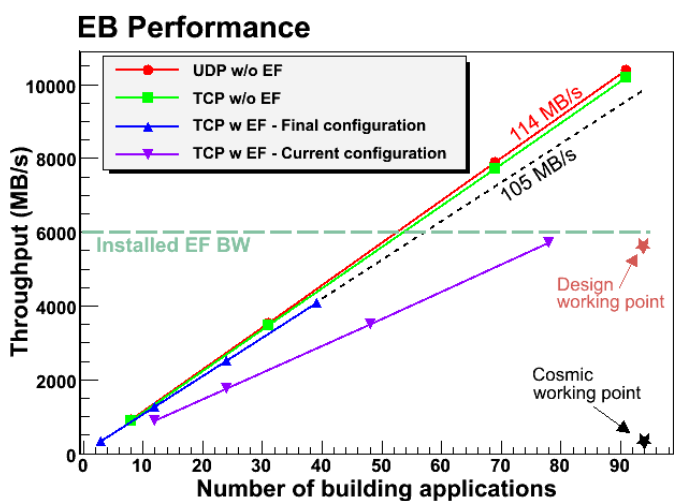

Figure 2. EB performance, with and w/o EF, with TCP and UDP network protocols.

\section{The Current TDAQ System}

The ROS, EB and SFO sub-systems have been fully installed, according to the design specifications of 153, 63 and 6 nodes respectively. The HLT farm is currently made of 850 nodes, 35\% of the expected final system. These nodes can be configured as either LVL2 or EF and typically, in early 2010 data taking, 250 are used for LVL2 and 600 for EF, running a total of 2000 LVL2 and 4800 EF processes. Further nodes are going to be installed in the next months.

The SFO farm consists of 6 nodes, 5 for production plus 1 hot spare, with a storage capacity of 10.5 TB/node. An SFO holds 3 RAID-5 disk controllers, each supervising 8 disks, in order to maximise the bandwidth. The sustained rate is $550 \mathrm{MB} / \mathrm{s}$, with peaks of more than $700 \mathrm{MB} / \mathrm{s}$. The total capacity allow buffering for at least two days of data, in case of central storage or network failure.

Comparing the software requirements at design and now, there are also major differences to be noted. The design foresaw $\mathrm{O}(1 \mathrm{k})$ software processes for the whole control tree. Currently there are $\mathrm{O}(10 \mathrm{k})$ software processes controlling the system. This is due to the multicore technology, unforeseen at the time of design, resulting in one process running on every core. A Finite State 
Machine (FSM) stears the system. The FSM was supposed to provide a fast start and stop for a change of run, and to configure to load new settings. With the highly increased number of processes, the state transitions are presently slow, and moving the full system to new conditions takes more time than foreseen. In average, the start transition needs about $110 \mathrm{~s}$, the stop $170 \mathrm{~s}$, and the configure even $260 \mathrm{~s}$.

Events are currently rejected as late (and as little) as possible, to allow the commissioning of the triggers. With just few $\mathrm{kHz}$ of $\mathrm{LVL} 1$ rate, LVL2, EF and SFOs are working near and sometime beyond the design throughput specifications. Finally, the 1.6 MB average event size is slightly reduced to $1.5 \mathrm{MB}$ due to the partial event building contribution. The system is also able to successfully handle a large variety of event sizes (up to almost $5 \mathrm{MB}$ per event), as determined by specific sub-detector needs.

\section{Data Taking}

In order to maximise the data taking efficiency during the not yet regular LHC working cycle, automatic procedures for a fast and reliable error recovery, as well as for a fast start-up, have been put in place:

- stopless removal and recovery

- dynamic resynchronisation

- warm start/stop

The stopless removal mechanism allows to remove faulty elements blocking the trigger completely, without having to stop the run. It is followed by a dynamic resynchronisation with the LHC clock and by the reinsertion in the readout of the faulty elements. At this point, the expert system framework is automatically notified and takes care of re-enabling the channels. The dynamic resynchronisation can also be used as a preventive method, recovering a desynchronisation before a trigger stop has been provoked.

Not all the ATLAS components can be powered at the nominal voltage before the beams get into stable conditions. For example, the silicon pixel detector, located at few centimeters distance from the beam pipe, could be seriously damaged by astray proton radiations. With data-taking ongoing, once stable beam condition has been established, the expert system automatically triggers the "warm start" procedure. All the detectors are eventually brought to nominal working conditions by the operators. The expert system then automatically set the trigger configuration according to the desired physics menu. A similar procedure is used for the "warm stop" in case the beams become unstable.

The live-time efficiency, defined by the presence of both stable beams and ATLAS data taking, is calculated as follows:

eff $=\frac{T_{\text {RunBeam }}-T_{\text {DeadTime }}}{T_{\text {Beam }}}$,

where $T_{\text {Beam }}$ is the total period with colliding stable beams, $T_{\text {RunBeam }}$ the actual running time, and $T_{\text {DeadTime }}$ the time in which ATLAS is not taking data. The latter can happen due to several reasons, such as a change of trigger prescales or busy channels in the system.

Over the last 4 months, the overall efficiency has been of $\sim 96.7 \%$.

\section{Conclusions}

The ATLAS three-level trigger architecture make use of regions of interest to reduce the dataflow requirements. It is a highly distributed system over $\sim 3000$ nodes. One third of the highlevel trigger farm is installed, more machines are coming within the next few months. As the commissioning of the triggers is currently ongoing, events are rejected as late as possible, as opposed to the design. The level 1 trigger output rate is low, currently at $\mathrm{O}(1 \mathrm{kHz})$, due to the start-up mode operations. Automatic procedures for maximise the live-time are in place. The overall data taking efficiency is excellent.

\section{REFERENCES}

1. ATLAS Collaboration, The ATLAS Experiment at the CERN Large Hadron Collider, JINST 3 (2008) S08003.

2. The ATLAS Collaboration, ATLAS DAQ, EF, LVL2, and DCS, 1998 CERN/LHCC/9816 